\title{
The isochronal fibration: Characterization and implication in biology
}

\author{
Hedi Ben Amor * . Nicolas Glade* . \\ Claudio Lobos **. Jacques Demongeot *
}

Received: date / Accepted: date

\begin{abstract}
Limit cycles, because they are constituted of a periodic succession of states (discrete or continuous) constitute a good manner to store information. From any points of the state space reached after a perturbation or stimulation of the cognitive system storing this information, one can aim to join through a more or less long return trajectory a precise neighbourhood of the asymptotic trajectory at a specific moment (or a specific place) on the limit cycle, i.e. where the information of interest stands.

We propose that the isochronal fibration, initially imagined and described by A. T. Winfree may be an excellent way to connect directly those two locations. Each isochron is indeed the set of points in temporal phase with one single point of the attractor. The characterisation of the isochronal fibration of various dynamical systems is not easy and until now has principally only been done numerically but not analytically.

By integrating the homogeneous solutions of the dynamical system we can solve this fibration in the case of the well known anharmonic pendulum. Other isochronal fibration on classical examples such as the van der Pol system and the non-symmetrical PFK limit cycle are obtained numerically and we also provide the first numerical study on 3-dimentional systems like the anharmonic pendulum with a linear relaxation on its third variable and the Lorenz attrac-

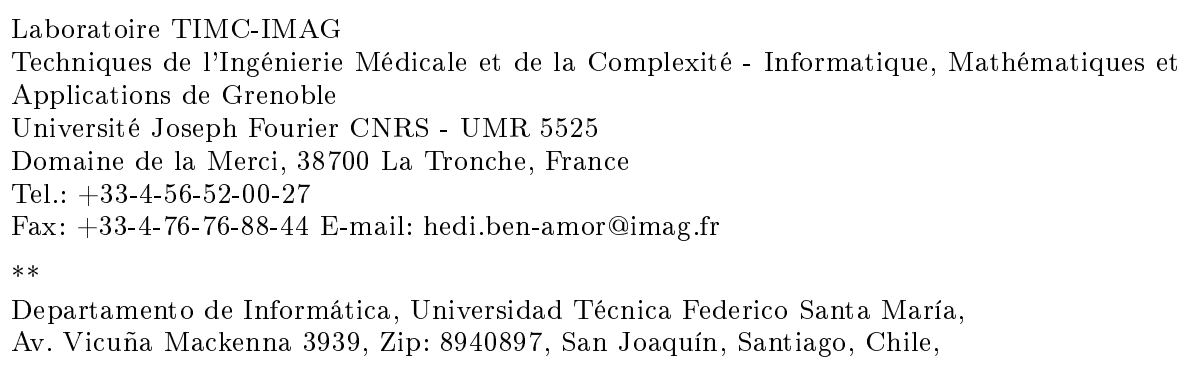


tor. The empirical approach seems us useful for dealing with the isochronal fibration which could constitute a powerful tool for understanding and controlling the dynamics of biological or biological-inspired systems.

Keywords Dynamical Systems · Isochrons - Mnesic Evocation · Isochronal Fibration · Trajectory · Attractor · Anharmonic Pendulum · PFK · Lorenz attractor

\section{Introduction}

Temporal persistence and spatial localization (indexation) are the 2 substrates essential to store information. A limit cycle is then a support offering these 2 aspects. In the state space of a dynamical system described from a set of particular initial conditions: a limit cycle is an example of attractor and the set of initial conditions converging to it forms its basin of attraction. Each point of the basin of attraction is associated to a latent asymptotic phase. This notion was introduced by Winfree (1974) and is defined as follows: the latent phase of a point in the state space is equal to the phase on the immediate neighbourhood of the attractor after a sufficient time, equal to a number of periods $\mathrm{T}$ ( $\mathrm{T}$ being the period of revolution of the limit cycle). The set of points having the same latent phase is called isochron (Winfree, 2000). In other terms, an isochron looks like the set of the successive observations performed during a stroboscopic experience. A method developed by Minorsky (1959) is based on the same principle. It is called the stroboscopic method. It consists on transforming the differential equation (expressed on polar coordinates) into a stroboscopic differential equation. Despite the fact that this method is a way to characterize isochrons, Minorsky did not takle an equivalent notion of isochron as Winfree did. Then, every point of the limit cycle is associated to a hypersurface called 'isochronal fibration' (Winfree, 2000). Figure 18 illustrates this notion. An isochron has the aspect of a one dimensional fibre when the state space is bidimensional, and of a surface (membrane) when the state space is 3 -dimensional. It's dimension is $n-1$ when the state space is a n-dimensional space.

Historically, the first practical use of the notion of isochron has been done for interpreting the responses of a population of coupled biological oscillators to periodic stimulations, like the oscillators involved in the photosensitivity of the circadian time-sense in Drosophila pseudoobscura (Winfree, 1967) and the first introduction of that notion in respiratory physiology concerned the entrainment of the respiratory rhythm in cat (Dinh et al, 1983).

Therefore, isochrons allow us to have an idea about the dynamics of the system in terms of velocity of trajectories. For example, in a bottleneck area of isochrons, the trajectories tend to have a slow velocity. On the other hand, knowing the isochrons of a dynamical system allows us to predict its future state after being perturbated, thus anticipating its return to the periodic limit 
cycle $^{1}$. We enhance this analysis by a measurement of the synchronization response to perturbation in a population of homogenous oscillators. This allows us to classify the phase space into fast an slow synchronization regions. The determination of isochrons is generally done via numerical methods. This is due to the difficulty (coming from the absence of homogenous solution of the system) to solve them analytically. Demongeot et al. (Demongeot et al, 2007b,a; Glade et al, 2007; Forest et al, 2007) infer an analytical approximation of the isochrons based on a potential-Hamiltonian decomposition. The authors suggest that the isochronal fibration tends to fit the potential part of the flow when the Hamiltonian part is dominant, particulary in the neighbourhood of the attractor. Thus, in (Demongeot and Françoise, 2006), the authors propose an approximation at the first order of the isochrons of polynomial Hamiltonian systems. Recently, Osinga and Moehlis (Osinga and Moehlis, 2009) proposed a very accurate method for approximating the global isochrons. The method is based on the continuation of a two-point boundary value problem and then of the linear approximation of the isochrons in the close neighbourhood of the attractor.

In the present article, we focus more on the use of isochrons in the understanding of biological systems showing periodic behaviours. Limit cycles appear indeed frequently in numerous biological systems, particularly in neural networks but also in many regulatory networks. It has been notably demonstrated that the asymptotic behaviours of neural networks is constituted by limit cycles (over large ranges of parameters) in the case of discrete (Dinh et al, 2008) and continuous (Budelli et al, 2010) -time (and continuous-space) models. It is also verified in real neural networks (Reyes, 2003). In the general case of discrete time and space models (like in Hopfield-like networks), the proportion of systems showing limit cycles increases with the number of nodes (Elena et al, 2008; Demongeot et al, 2009; Ben-Amor et al, 2009; Elena, 2009).

We characterize analytically the isochrons of the anharmonic pendulum by integrating its homogenous solutions. We give examples of the isochronal fibration of some 2-dimensional systems either symmetrical (such as the van der Pol system or the Wilson-Cowan system) or non-symmetrical (such as the PFK system). We also calculated the isochronal hypersurfaces of 3-dimensional systems in the case of an anharmonic pendulum having an additionnal linear relaxation in the third direction, and in the case of the Lorenz attractor. In the latter, we looked at their shapes in the state space by a series of cuts and $3 \mathrm{D}$ reconstructions by triangularisation methods. Finally, we discuss on their importance, notably in the understanding of desynchronisation in cyclic biological processes such as those involved in the glycolysis (the PFK F6P/ADP induced periodic behaviour) or in the neuronal activity that occurs during mnesic evocation.

1 If we do know in which isochron the state of the perturbed system is then we know in which point he will join the attractor 


\section{Mathematical definition of an isochron}

Let us consider the space $E \subset \mathbb{R}^{n}$ of all states of a dynamical system and the times set $T \subset \mathbb{R}$ (containing time 0 ). A flow $\varphi$ is an application from the Cartesian product $E \times T$ onto $E$ satisfying the following properties: $\forall x \in$ $E, \forall s, t \in T$, then: (i) $\varphi(x, 0)=x$, (ii) $\varphi(\varphi(x, t), s)=\varphi(x, t+s)$. The implicit notation of the flow $x(t)$ which stands for $\varphi(x, t)$ is called trajectory. The initial condition is expressed by $x(0)=x$. We stress that the value $x(t)$ is the state of the dynamical system at time $t$ such that its state at time 0 is $x$. The orbit of a state $y$ iterated by the flow $\varphi$ is the set of points $\mathcal{O}_{\varphi}(y)=\{\varphi(y, t), t \in T\}$. We define the Birkhoff limit set $L(x)$ of any state $x$ of $E$ taken as intial condition of $x(t)$, as the set of temporal accumulation points of this trajectory. If $B(y, \varepsilon)$ denotes the set of states at distance of $y$ less than $\varepsilon(B(y, \varepsilon)$ is reduced to $\{y\}$ in the discrete case): $L(x)=\{y \in E ; \forall \varepsilon>0, \forall t \in T, \exists s \in T / s>t$ and $\left.\mathcal{O}_{\varphi}(\varphi(x, s)) \cap B(y, \varepsilon) \neq \emptyset\right\}$

The attractor basin $B(A)$ of a subset $A$ of $E$ is the set of all initial conditions $x$ not in $A$, but such as $L(x) \subset A$.

By introducing the necessity to exlude $A$ from $B(A)$, we eliminate the possibility to designate by attractors the closed trajectories of a Hamiltonian system (like the Lotka-Volterra).

Let us denote $L(A)=\cup_{x \in A} L(x)$. $\bar{A}$ is the set $A$ completed by all possible shadow trajectories (Bowen, 1975). An attractor $A$ verifies (Cosnard and Demongeot, 1985b,a):

i) $A$ is a fixed set for the composed set operator $L \circ B: A=L(B(A))$,

ii) there is no set $C$ such as $A \subset C \subset \bar{A}, C \neq A$, verifying i),

iii) there is no set $D \subset A, D \neq A$, verifying i) and ii).

An attractor $A$ is invariant in the dual operations consisting firstly in considering all the trajectories of its basin from all initial conditions not in $A$, but finishing their life in $A$, and secondly to restrict them to their ends of life (Figure 1).

The notion of shadow trajectories allows to include inside an attractor all the points at discrete distance 0 from the attractor. For example, the Hamming distance can be relaxed to a quasi-pseudometric. We can consider that below $k$ different bits the distance between two sequences of bits is 0 . This is defined by the quasi-pseudometric $d_{H_{k}}(x, y)=\operatorname{Max}\left(0, n_{\text {diff }}-k\right)$, where $n_{\text {diff }}$ is the number of different bits. Note that $d_{H_{0}}$ corresponds to the Hamming distance. When quasi-pseudometrics are used, one throw the axioms of the identity of indiscernibles, i.e. $d(x, y)=0$ for some $x \neq y$, and the triangular inequality is not verified due to a change of behaviour of the distance function depending on the sign of $n_{\text {diff }}-k$ (rough-grain below $k$, fine-grain otherwise).

Let us suppose now that the attractor $A$ is a limit cycle, i.e., if we denote by $p$ the period of the limit cycle, $A=\left\{a_{0}, a_{1}=\varphi\left(a_{0}, 1\right), \ldots, a_{p-1}=\varphi\left(a_{0}, p-1\right)\right\}$ and there is a natural isomorphism $\psi$ between $A$ and the set $S=\{0, \ldots, \tau-1\}$. By denoting $T_{s}=\{t \in T / t=s+k \tau\}_{k \in N, s \in S}$ we have: $T=\cup_{s \in S} T_{s}$.

The isochron $I_{s}$ of phase $s$ is the attractor basin of $\left\{\psi(s)=a_{s}\right\}$ for the flow 
$\varphi_{s}$ (equal to $\varphi$ on $E \times T_{s}$ ) and $B(A)=\cup_{s \in S} I_{s}$. If $T=\mathbb{R}+$ (the positive real numbers set), $I_{s}$ is transversal to $A$, i.e., the tangent vector to $I_{s}$ at the state $a_{s}$ is not tangent to $A$ (Guckenheimer, 1975; Freire et al, 2007).

\section{Numerical resolution of isochrons}

All points delayed by a period $T$ (period of the limit cycle) are on the same isochron because they have the same latent phase ${ }^{2}$. The determination of these points can be done by using numerical methods. Whatever the numerical method used is, checking if points of the state space belong to the isochron of a chosen phase can be obtained by verifying, when they converge until the attractor via the equations of the dynamical system, if they admit that phase of the attractor once considered a certain limit. Several numerical methods are possible: a systematic exploration of the state space by dividing it in small areas, a random exploration in all the state space, or a local random exploration with a guidance of the research area along a direction given by the points already founded (a kind of intelligent paintbrush). We pull out a point of the state space randomly, then we compute a discrete version of the differential equations until the trajectory reaches for the first time a point belonging to a neighbourhood of fixed thickness $10^{-k}$ of the attractor, where $k$ is a thickness parameter. The phase of this point on the attractor is memorized. This phase minus the phase of the nearest isochron of interest is compared to a tolerance value in order to determine whether this point can be considered as belonging on the isochron or not. This tolerance value is equal to $\frac{T}{2 n p}$ (figure 2) where $T$ is the period of the attractor considered as a limit cycle, $n$ is the number of isochrons considered and $p \in[1,+\inf [$ is a precision parameter.

\subsection{Bidimensional systems}

\subsubsection{Symmetrical and anti-symmetrical systems}

In 2-dimensional systems, isochrons of symmetrical and antisymmetrical systems (characterized by the shapes of their limit cycles) are easily obtained and their isochrons correctly numerically resolved, on the contrary to nonsymmetrical systems (see below). The anharmonic pendulum (Demongeot et al, 2007b,a; Glade et al, 2007; Forest et al, 2007), which is a particular $\lambda \omega$-system (Murray, 1993), is a typical case of symmetrical system whose isochrons are radially disposed. This system is studied in section (5). The Van der Pol oscillator shown in Figure 3 (Right) is defined by the differential system $d x / d t=y, d y / d t=-x+\mu y\left(1-x^{2}\right)$ and $\mu=2$. The resolution of its isochrons needs a very important precision in areas of convergence of multiple

2 However, an isochronal fibration is continuous, figure 18 illustrates this. 
isochrons (i.e., at the neighbourhood of the phaseless point $(0,0)$ ) and on the right and on the left sides of the attractor (Figure 3 Right).

\subsubsection{Non-symmetrical systems: the example of the Phosphofructokinase dynamical system}

In Ricci (1995), a dynamical system is given modelling the PFK (Phosphofructokinase) behaviour. It describes the balance between the consumption of the substrate, the F6P (Fructose6-phosphate), and the production of the product, the ADP (Adenosine Diphosphate). It is defined by $d x / d t=A-L, d y / d t=$ $R(L-N y)$ with $L(x, y)=\frac{x\left((1+x)^{n-1}(1+D y)^{n} L_{0} C(1+C x)^{n-1}\right)}{\left((1+x)^{n}(1+D y)^{n}+L_{0}(1+C x)^{n}\right)}$ and $R=10^{P}$. The numerical determination of the isochrons of the PFK was obtained for the following values of parameters: $L_{0}=3000, n=3, C=0.02, D=1, A=0.1$, $N=0.01$ and $P=3$. The PFK system exhibits a non-symmetrical limit cycle with a region of the attractor where velocity of the flow is very fast. This behaviour makes the resolution of the isochrons of the system hard to perform. Winfree (2000) wrote: "In practice, the isochron structure is poorly resolved near the isochrons' convergence... That implies unusual difficulty in measuring phase with adequate precision near the region of convergence. It also means that the system is particulary susceptible to the random perturbations that chronically afflict any real experiment, especially biological experiments". This is indeed what Figure 4 clearly shows. We tested several values for the precision parameter between 1 and 1000. The two extremes we calculated are shown with the lowest precision (1) on the left and a highest precision (1000) on the right. We calculated here 30 isochrons, which appear very tightened in some regions of the state space. Moreover, at the bottom of the 2 figures appears a region where isochrons seem to be overlapped and poorly resolved even at high precisions. This means that, at a given resolution, latent phases are not so clearly allocated to their isochrons, so that a certain synchronisation is lost. Theoretical isochrons of 2-dimensional systems are unidimensional fibres, but 'real ones', i.e., those determined by numerical methods (resp. measured from experiments on natural systems) are very sensitive to numerical errors (resp. natural noise or experimental imprecision) in such desynchronisation areas.

\subsection{Tridimensional systems}

The isochrons of 3-dimensional systems are 2-dimensional surfaces. We used the same algorithm as for 2-dimensional systems. When a valuable density of solved points is reached (e.g., about $4.10^{6}$ points for the Lorenz pendulum), the algorithm stops. Such a huge number of points is hard to handle and to visualize ${ }^{3}$, so we used meshing techniques to reconstruct the surfaces and render them. Moreover, cuts in the state space and through the isochronal

\footnotetext{
3 exploration (rotations, zooms, ...) and visual rendering (lights and shadows) of the surface
} 
surface allow to have a better understanding of their complicated shapes. Two systems are presented here: the anharmonic pendulum-like system and the Lorenz system.

\subsubsection{Anharmonic pendulum-like system}

We add a third component to the classical anharmonic pendulum. The $3^{\text {rd }}$ component is a linear relaxation to the plane $(x y)$. This is expressed by the following differential system: $d x / d t=y+x\left(1-x^{2}-y^{2}\right), d y / d t=-x+y\left(1-x^{2}-y^{2}\right)$ and $d z / d t=-k z$ with $k>0$. As the relaxation is linear and normal to the attractor, the isochrons of this system are intersecting half-planes in the $z$ axis. The isochrons of this system are presented in Figure 5.

\subsubsection{Lorenz system}

The strange attractor of Lorenz has been a main step of the chaos theory. The system is defined by the differential system: $d x / d t=\sigma(y-x), d y / d t=$ $\rho x-y-x z$ and $d z / d t=x y-\beta z$. We calculated its isochronal fibration within conditions $(\sigma=10, \rho=350, \beta=8 / 3$ ) giving a periodic behaviour (a periodic attractive orbit numerically resolved) of period $T=0.37$ and only one loop, instead of a chaotic one. Isochrons are continuous surfaces in the 3-dimensional state space (Winfree, 2000). They appear as very complicated surfaces with quasi-planar parts and regions of strong curvature (Figure 6). We also observed that a certain desynchronisation occurs in some regions where the isochrons are very close together and where overlaps are observed.

\section{Analytical resolution of the isochrons of the anharmonic pendulum}

Isochronal fibration can be described by another approach based on a temporal discretization of the initial system by choosing as a time step the period $\mathrm{T}$ of revolution of the system. In other words, the flow of the new system will obtained by integrating on the time set $T_{s}$ (where $\mathrm{s}$ is the phase of the isochron) the velocity of the studied dynamical system. The obtained flow will help us to obtain the position of the points having the same phase (therefore being on the same isochron) until their convergence to the corresponding point in the attractor. The expected value of the distance between successive points on the isochron will tend to zero when the number of iterations tends to infinity.

In the following, we have studied the isochrons of the anharmonic pendulum. This system is advantageous: it is simple, the expression of its homogeneous solutions is known and its isochrons are in the simpliest expression: they are radially distributed. The differential equations that describe it are: $d x / d t=y+x\left(1-x^{2}-y^{2}\right), d y / d t=-x+y\left(1-x^{2}-y^{2}\right)$ and their homogeneous solutions (described in (Demongeot et al, 2007a)) are: $x(t)=\rho_{0} e^{t} \frac{\cos \left(t-\theta_{0}\right)}{\sqrt{1+\rho_{0}^{2}\left(e^{2 t}-1\right)}}$, 
$y(t)=-\rho_{0} e^{t} \frac{\sin \left(t-\theta_{0}\right)}{\sqrt{1+\rho_{0}^{2}\left(e^{2 t}-1\right)}}$ with $\left(\rho_{0}, \theta_{0}\right)$ the polar coordinates corresponding to $(x(0), y(0))$.

Let $\psi$ the limit cycle of the system. Integrating on a strictly positive number $k$ of revolutions on the attractor $\psi$ is formalized by: If $(x(0), y(0)) \in \psi$ then $\forall k \in N^{*} \int_{0}^{k T} \frac{d x}{d t} d t=\Delta_{0}^{k T} x=0$ and $\int_{0}^{k T} \frac{d y}{d t} d t=\Delta_{0}^{k T} y=0$ with $T>0$.

Then, we solve the system $\Delta_{0}^{k T} x=\rho_{0} \frac{\cos \left(k T-\theta_{0}\right) e^{k T}}{\sqrt{1+\rho_{0}^{2}\left(e^{2 k T}-1\right)}}-\cos \left(\theta_{0}\right)=0$ and $\Delta_{0}^{k T} y=\rho_{0} \frac{\sin \left(k T-\theta_{0}\right) e^{k T}}{\sqrt{1+\rho_{0}^{2}\left(e^{2 k T}-1\right)}}-\sin \left(\theta_{0}\right)=0$ with $T>0$.

As a first approximation, we will suppose that $\rho_{0}=1$ and $\theta_{0}=0$ is a point belonging to the attractor, so we have $T=2 \pi$.

Let us notice that the two entities $\Delta_{0}^{T} x$ et $\Delta_{0}^{T} y$ represent the respective variations of $x$ and $y$ after one revolution ${ }^{4}$. So, $x(T)=x(0)+\Delta_{0}^{T} x$ and $y(T)=y(0)+\Delta_{0}^{T} y$. Therefore, let us define $x_{n+1}=x(T), x_{n}=x(0)$ and we apply the same for $y$. By substituting in $\Delta_{0}^{T} x$ (resp. $\left.\Delta_{0}^{T} y\right) \rho_{0} \cos \left(\theta_{0}\right)$ (resp. $\left.\rho_{0} \sin \left(\theta_{0}\right)\right)$ by $x_{n}$ (resp. $y_{n}$ ) and $\rho_{0}^{2}$ by $\left(x_{n}^{2}+y_{n}^{2}\right)$, we obtain the following discrete system: $x_{n+1}=x_{n} * \frac{e^{2 \pi}}{\sqrt{1+\left(x_{n}^{2}+y_{n}^{2}\right) *\left(e^{4 \pi}-1\right)}}$ and $y_{n+1}=y_{n} * \frac{e^{2 \pi}}{\sqrt{1+\left(x_{n}^{2}+y_{n}^{2}\right) *\left(e^{4 \pi}-1\right)}}$.

It is formed by the series of points on the same isochrons starting from equally phase distributed initial points $\left(x_{0}, y_{0}\right)$ far from and around the attractor (Figure 7).

\section{Bridges over the attractors: Guckenheimer's effect}

In the very close neighbourhood of the attractor, the Hamiltonian part of the flow becomes dominant (and the trajectories appear like an onion structure), so the isochrons tend to fit the potential part of the flow (Demongeot et al, 2007b,a; Glade et al, 2007), i.e., they cross their attractor transversely (perpendicularly), as first described by (Guckenheimer, 1975). Nevertheless, we were wondering on how the isochrons that appear - from a macroscopical point of view - very tilted compared to the direction of the asymptotic trajectory (for example see the isochrons of the van der Pol system in Figure 3 or those of the PFK system in Figure 4), cross their attractor. We ran simulations to solve numerically, with a very high precision ( precision $\geq 10^{7} \Rightarrow$ tolerance $\rightarrow 0$ ), 2 isochrons of the van der Pol system (its isochrons are not perpendicular to the limit cycle like those of the anharmonic pendulum) in the very close neighbourhood of the limit cycle. We did not expect what we observed and what we consequently called the Gukenheimer's effect : isochrons form a particular structure having a crescent shape (or a kind of bridge), that locally breaks the monotony of the isochronal fibre (figure 8 ). The crescent shaped isochronal fibre crosses perpendicularly the limit cycle, even if the whole fibre (farest from the limit cycle) is stongly tilted compared to the asymptotic trajectory (Figure 8 right). We also looked at other systems like the Lorenz system and the

4 These entities are null when $\rho_{0}$ tends to 1 . The attractor of the anharmonic pendulum is indeed the unit circle. 
behaviour of the isochrons at the neighbourhood of the attractor is similar. It verifies the assumptions proposed in (Demongeot et al, 2007b,a; Glade et al, 2007; Demongeot and Françoise, 2006) and it is in agreement with Guckenheimer's assertions (Guckenheimer, 1975).

\section{Maximum phase shift computation}

Once considered a population of uncoupled oscillators of same nature, it may be of interest to have a measure of the synchronization that occurs after a perturbation tranlating the state of all the oscillators to an other region at the same time, i.e. conserving that way the shape of the limit cycle (see Figures 14 and 15). The isochronal fibration gives a qualitative idea of the phase shift or on the contrary of the resynchronization which may result from a perturbation affecting this population. The divergence (resp. convergence) of equally distributed isochrons in a particular region of the phase space means indeed that this region is a fast (resp. slow) synchronization one. A quantitative measurement can be calculated from this fibration. We computed the phase shift between the two isochrons containing the set of $n$ points $\left(P_{i}\right)_{i \in[1, n]}$ obtained after a translation of the limit cycle as follows:

- The phase $\phi_{i}$ of each $P_{i}$ is calculated as described in section 3 .

- The vector $\left(P_{i}\right)_{i}$ is sorted by order of the increasing values of $\phi_{i}$.

- The vector $\left(\left(\Delta \Phi_{i, i+1}\right)_{i \in[1, n-1]}, \Delta \Phi_{n, 0}\right)$ is determined. $\Delta \Phi_{i, i+1}=\phi_{i+1}-\phi_{i}$ for $i \in[1, n-1]$ and $\Delta \Phi_{n, 0}=2 \pi-\left(\phi_{n}-\phi_{0}\right)$.

- The maximum phase shift is $\Delta \Phi_{M a x}=2 \pi-\operatorname{Max}\left(\left(\Delta \Phi_{i, i+1}\right)_{i \in[1, n-1]}, \Delta \Phi_{n, 0}\right)$.

Those steps are repeated for several values of the amplitude between 0 and $R_{\max }$ (maximum perturbation). When the system is symmetrical, we compute the maximum phase shift along an arbitrary direction of perturbation 13 . This is not valid when the dynamical system is asymmetrical like the phospho-fructo kinase (PFK) system (see Figure 9).

\section{Mnesic Evocation}

Let us consider a network (neural or genetic) made of several subsystems, like modules, identical or different, and weakly or strongly connected (Elena et al, 2008; Demongeot et al, 2009) : in Figure 10, M1 is an arbitrary subnetwork and M2 is made of a simplified Hippocampus one-layered network, with one Cyto-Architectural 1 (CA1) and one Cyto-Architectural 3 (CA3) pyramidal neuron, one Entorhino-Cortical neuron (EC) and one Inter-Neuron (IN), all interconnected. If we simplify this module in a subnetwork of size 2 , we obtain a structure called negative regulon, with one negative circuit and 2 positive self-loops. We will consider in the following that this subnetwork is sequentially repeated in a chain of modules, each of them being weakly linked to the 
following at the level of the CA3 neurons (the $X_{i}$ 's in Figure 11): CA3 neurons send axons with excitatory synapses to CA1 and CA3 neurons, these latter sending axons with excitatory synapses to EC neurons through the Subiculum (SB), and EC forming global inhibitory connections with CA3 neurons through Inter-Neurones (IN) of Dentate Gyrus (DG) (Buzsaki, 1984; Gluck, 1996; Hefft and Jonas, 2005; Bartesaghi et al, 2006; Mori et al, 2007).

When the system WC is stimulated through a perturbation $S$ translating all the initial conditions on the limit cycle $C$ into a fast region (Figure 14), then all the return trajectories go to the same phase $x_{1}$, provoking a synchronization of all the activities $X_{i}$ 's. In other terms, if the $X_{i}$ 's before the stimulation are dispatched uniformly on the limit cycle $C$, then their sum denoted $A(t)=\sum_{i=1, \ldots, n} X_{i}(t)$ is about zero, because the positive values of the $X_{i}$ 's are compensated by the negative ones. If the $X_{i}$ 's run in phase, $A(t)$ behaves like $n X_{i}(t)$ and can be detected for example by a Pet-scanner of a functional MRI device (Figure 15). The Wilson-Cowan system WC (Wilson and Cowan, 1974) used to simulate the dynamics of a subnetwork represented by a negative regulon (Figure 11) is given by $2 n$ differential equations: $\forall i=1, \ldots, n, d X_{i} / d t=-X_{i} / a+\tanh \left(b X_{i}\right)-\tanh \left(b Y_{i}\right)+k X_{i-1}, d Y_{i} / d t=$ $-Y_{i} / a+\tanh \left(b X_{i}\right)+\tanh \left(b Y_{i}\right)$. This system is closed to a Hopfield neural network, when $b$ is positive and large, and $a$ is negative and large (Tonnelier et al, 1999) whose dynamics were fully characterized in (Elena et al, 2008; Demongeot et al, 2009). Trajectories and isochrons of WC are represented in Figure 12, showing fast (resp. slow) regions, i.e., zones where the flow runs fast (resp. slow).

If the coupling between the WC subnetworks is made at the level of the CA3 neurons (whose activities are the $X_{i}^{\prime}$ 's) is weak, then a desynchronization occurs (Figure 16-1), allowing the exit out the perseveration behaviour (Figure 16-2). If the neuronal activity is noised, the desynchronization obtained with a weak coupling is not perfect (Figure 16-3), as well as the perseveration (Figure 16-4), both behaviours exhibiting a small residual synchronized activity. In case of synchronization, the global activity $A(t)$ of the CA3 neurons "evokes" the common limit cycle (Csicsvari et al, 1999) of the $X_{i}$ 's and can be considered as the phenomenologic result of the recall of a mnesic temporal pattern stored in the network (Demongeot et al, 2000; Samsonovich and Ascoli, 2005). If the succession of states to be transiently evoked is only a part of this attractor, then a fast desynchronization is needed, which is ensured by a weak coupling (Figure 17). The richness of the stored souvenirs comes from the number and the complexity of the common attractors of the $X_{i}$ 's. If their number increases (e.g., from 1 to 2 ) as well their complexity (e.g., passing from the circular limit cycle coming from a fixed configuration of focus type through a Hopf bifurcation, to a chaotic behaviour through doubling period bifurcations), then we can locally store and evoke complicated temporal patterns coding for complex cognitive entities or serving for moving objects detection in a complex scene (Hayashi et al, 2007). Such a bifurcations landscape can be simply obtained from the simulation of a fully connected Hopfield network of size 16, involved 
in a learning process, as shown on Figure 19 (Nérot, 1996).

\section{Conclusion}

By transforming the initial dynamical system, we have obtained a new discrete time system in which the trajectories are the isochrons of the first one. This new system has an infinity of fixed points and the set of those points constitutes the limit cycle of the anharmonic pendulum system. Therefore, one can imagine a linkage between those 2 systems in order to determine the phase of a perturbation and force the dynamical system to relax rapidly towards its attractor. For example, one can imagine 2 coupled oscillators (e.g., 2 coupled neural networks of regulon type (Glade et al, 2007) like in the cardio-respiratory system), the one having the behaviour of the anharmonic pendulum and the other having that of its 'isochronal system': then the switch from the one to the other would depend from the state - perturbed or not of the system, i.e., occurs when the distance between the isochronal fibres becomes greater than a certain limit. In this example, as for in neural networks applications like in mnesic evocation, the isochronal fibration constitutes a continuous phase and serves as synchrony set of pointers to the attractor. In the example of the anharmonic pendulum, we could have a direct expression of the isochronal fibration depending on the latent phase by integrating the homogenous solution of the initial system. However, most of the homogenous solutions of the dynamical systems are unknown. Therefore, it is difficult to apply this reasoning on other examples. Nevertheless, we think that it is possible to identify the dual dynamical system, 'isochronal system', by determining numerically the values of the latent phase $\phi$ and of the isochronal curvi-linear abscissa $\chi$ (equal to 0 on the attractor, positive out the limit cycle and negative inside), and by searching to match the 2 spaces $(x, y, t) \rightarrow(\chi, \phi)$. Finally, one can wonder if, as it is the case for the inner ear that physically treats the audio signals as a Fourier transform, our brain is able to work in 'isochron mode', i.e., to use these shortcuts. Future works on discrete Hopfield networks or continuous Wilson-Cowan networks shall try to implement (in the form of neural networks) such neuronal modules working in the isochron space $(\chi, \phi)$ coupled to other neuronal modules working in state and time space $(x, y, t)$. This would consitute a powerful tool for designing new storage systems and an advance in the researches on the memory and mnesic evocation. 


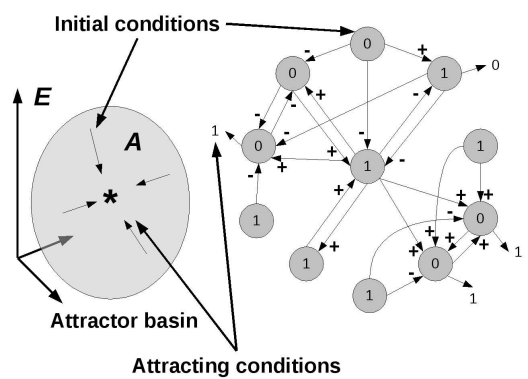

Fig. 1 Definition of an attractor (the character 'star') and its basin (the gray bubble) in the case of a Boolean network, whose the states space $\mathrm{E}$ is the hypercube $\{0,1\}^{11}$ (left). Initial conditions are indicated in gray disks and attracting conditions for the majority rule and synchronous iterations (the state of a node equals 1 if its activating neighbours in state 1 are equally or more numerous than the inhibiting ones) are given outside gray disks (right).

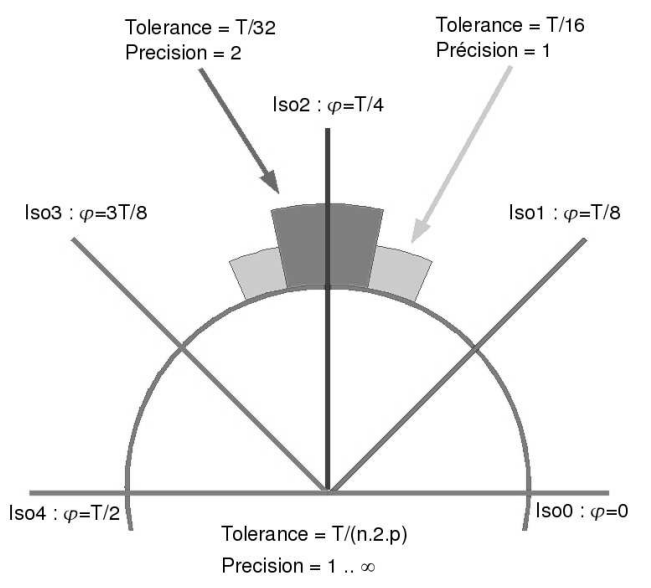

Fig. 2 Graphical description of the measure of precision Let us consider 8 isochrons equally spaced. The largest acceptable tolerance is $T / 16$, ie. the phase interval between 2 isochrons is equally divided. The precision parameter is used to express the tolerance parameter as follow: Tolerance $=T /\left(\right.$ Precision $\left.* 2 * N_{\text {isochrons }}\right)$ 


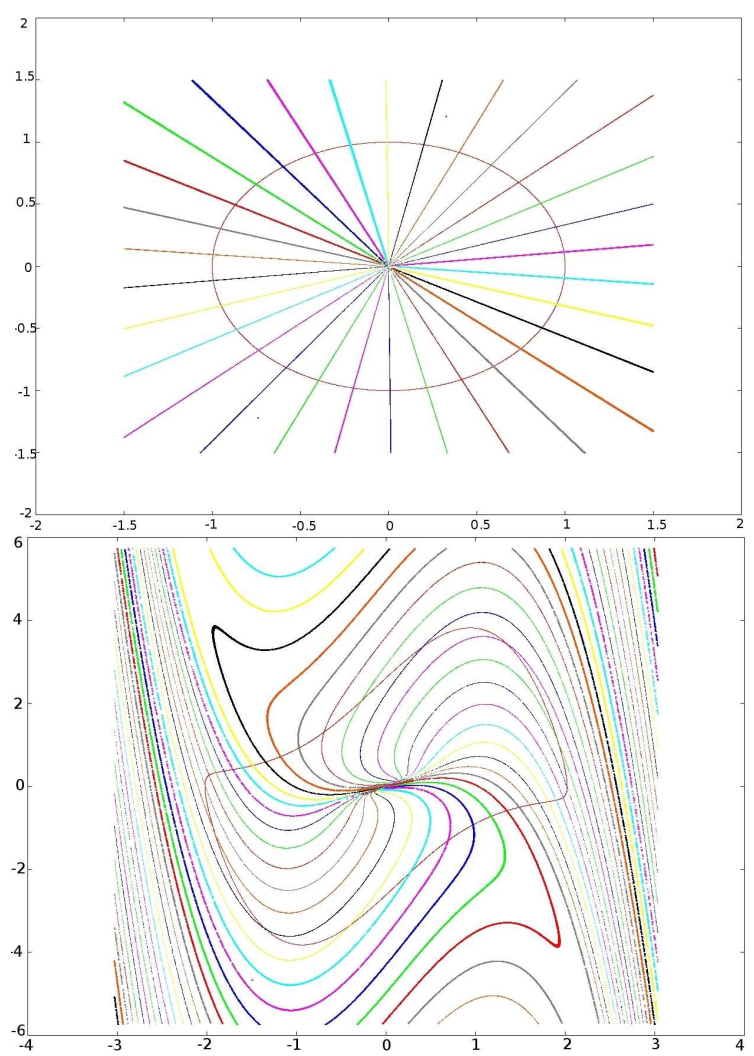

Fig. 3 Isochrons of different dynamical systems (Top) Radial isochrons of a symmetrical system: anharmonic pendulum. (Bottom) Isochrons of an anti-symmetrical system: the Van der Pol oscillator (parameter $\mu=2$ ). 


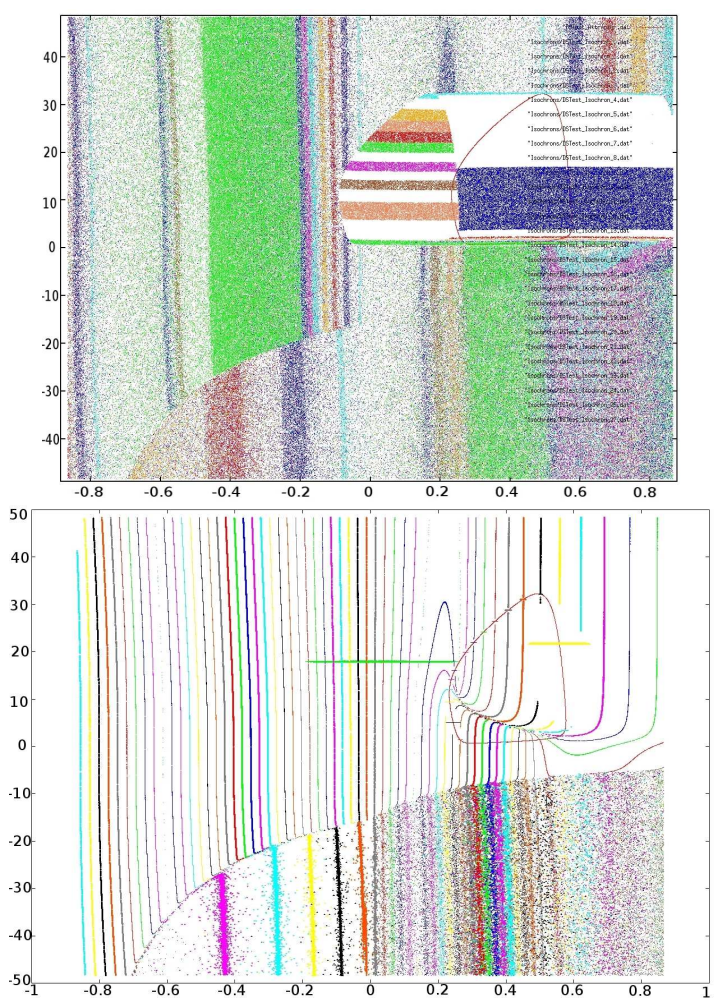

Fig. 4 Isochrons of an non-symmetrical system: the PFK enzymatic balance Isochrons and attractor of the PFK system obtained for (Top) a precision equal to 1 and (Bottom) a high precision (1000). Note that the area at the bottom of the two figures is high sensitive to numerical errors. In this area, the isochrons are overlapped, which indicates a sensitivity to numerical precision and corresponds to a desynchronisation area.

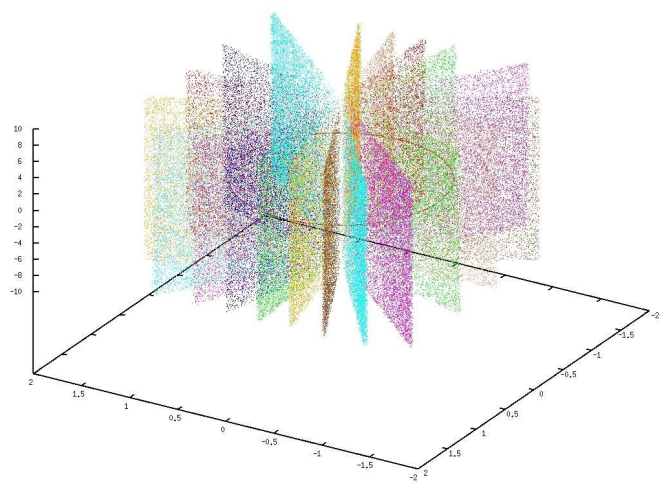

Fig. 5 Attractor and planar isochrons of the 3-dimensional anharmonic-like pendulum. 

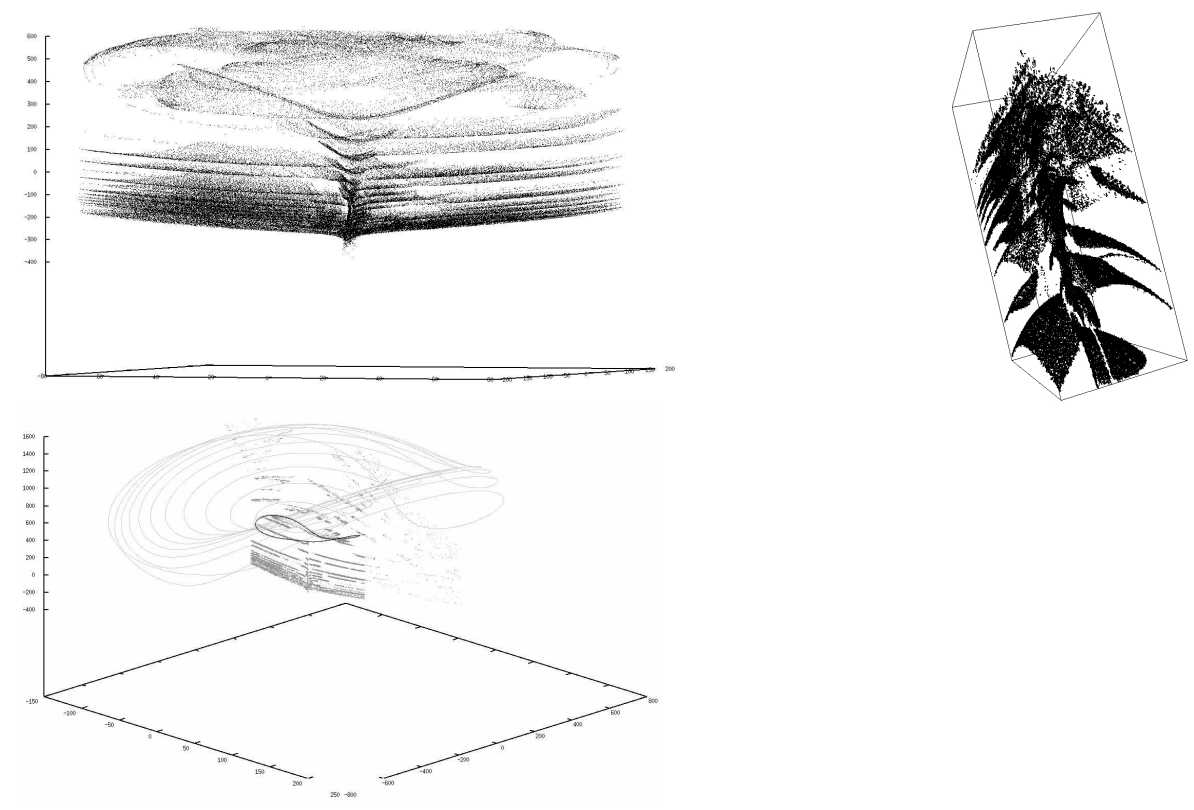

Fig. 6 One isochron of the Lorenz system (Top Left) Side view of one isochron of the Lorenz system and (Top Right) a reconstitution of its central part using meshing techniques. (Bottom Left) The attractor (black) is shown with a section ( $x z$ plane) of this isochron (dark grey) and a trajectory starting from a point of the isochron (light grey).
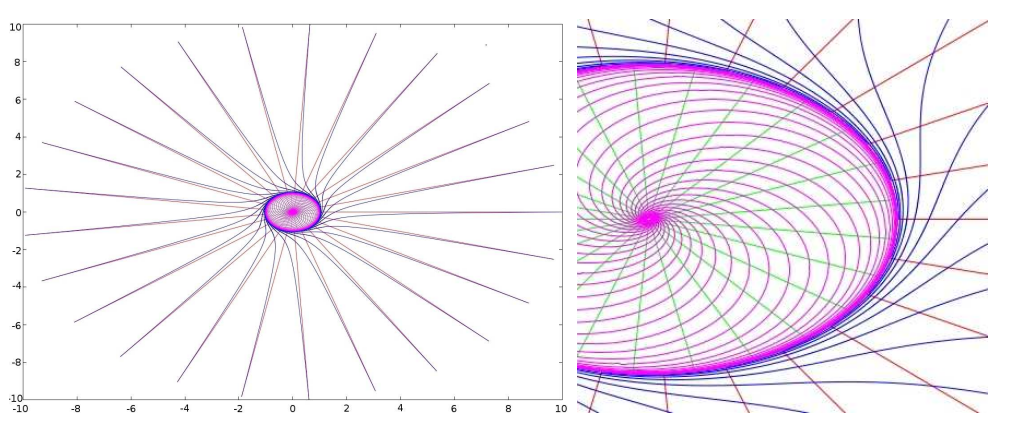

Fig. 7 Trajectories, attractor of the anharmonic pendulum and isochrons calculated from the discrete system given before (Left) The limit cycle of the system is circular and its isochrons are segments of straight lines. Far from and ouside the attractor, the system is mainly potential so the isochrons are asymptotes for the trajectories of the dynamical system (exponential part). (Right) In the area delimited by the attractor, the system is mainly Hamiltonian, so its trajectories are spring spiral curves. Here, the isochrons inside and outside the attractor are dephased by a half-phase. 

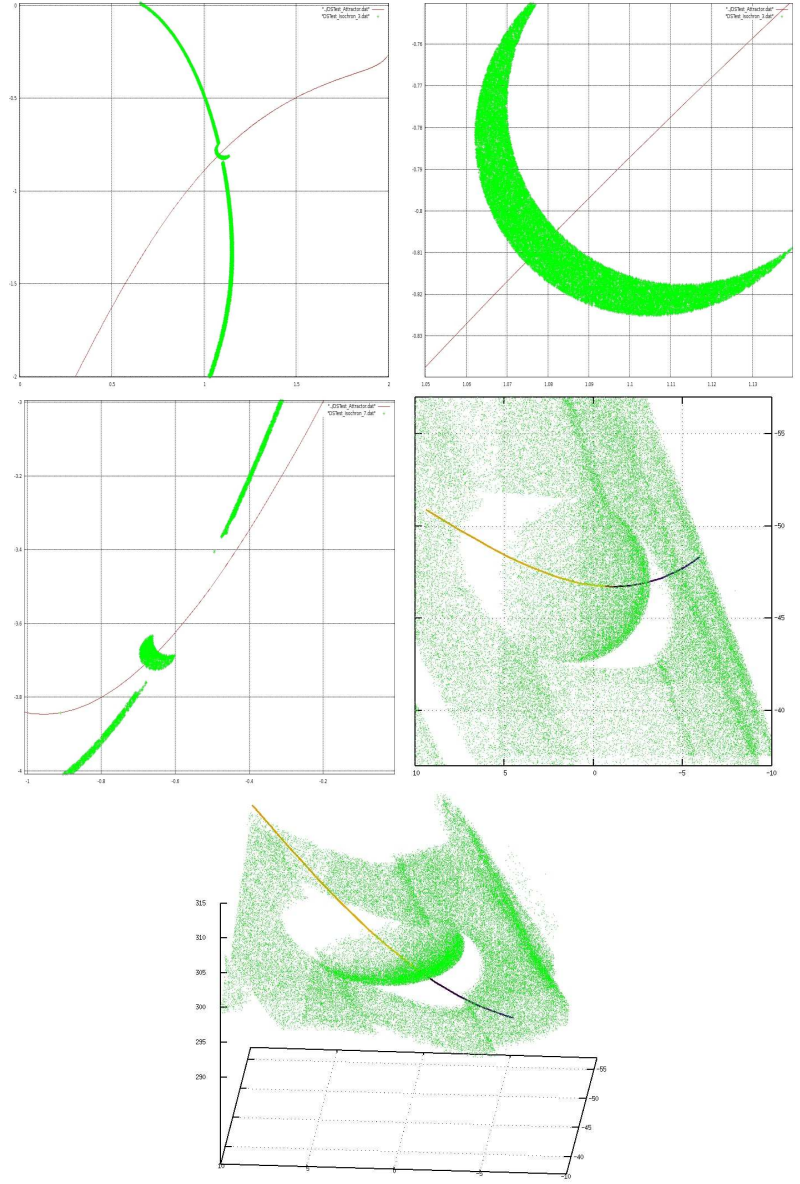

Fig. 8 Isochronal bridges over the van ver Pol attractor. (Top): Two isochrons (Left and Right) of the van der Pol system (see figure 3) are calculated with a very high precision. The picture in the center is a zoom of the crescent structure on the left. The isochrons on the right appear discontinuous. This is only due to a limitation of the numerical resolution. The same reason explains their thickness. (Bottom): The same is obtained in a 3-dimensional system, the Lorenz pendulum. The picture on the left is a top view of that on the right. 

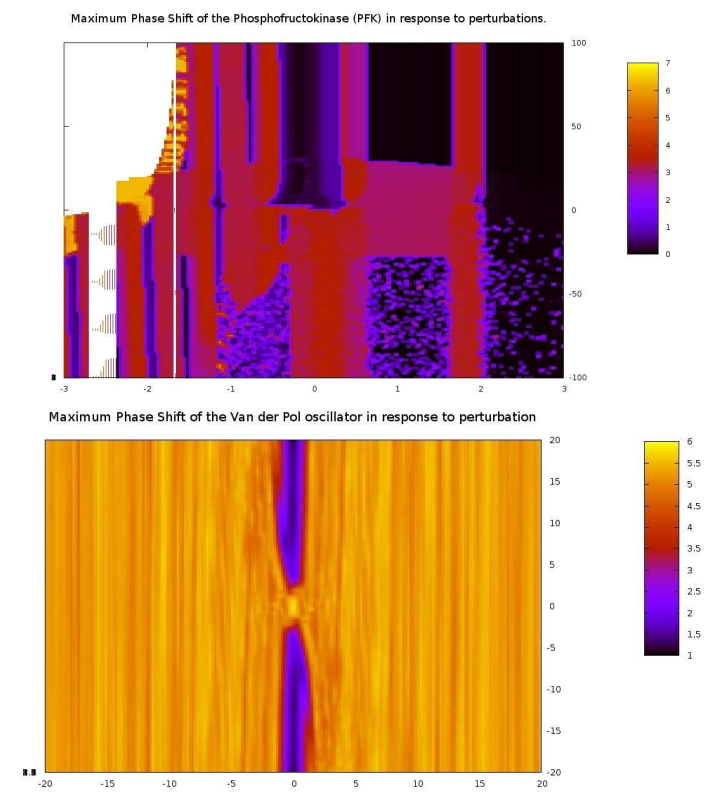

Fig. 9 Maximum phase shift of the PFK (Up) and the van der Pol (Down) oscillators in response to perturbations. Dark (resp. bright) colors correspond to fast (resp. slow) synchronization regions. White regions correspond to regions where the phase is not well-defined (very strong sensitivity to numerical imprecision). This synchronization map is very irregular and clearly shows that it is hard to sycnhronize the PFK (Up) due to a (strong non linearity and asymmetry of the system). Synchronizing the whole system needs to choose very precisely the direction and amplitude of the perturbations. This is very different for the van der Pol (Down) system where regions of synchronization (underneath and overhead) and desynchronization (on the right and on the left) are well defined and localized in the phase space. The synchronization map of the van der Pol system (Down) can be superimposed with the isochronal profile given in Figure 3 (down). One can observe the striking similarity between the slow (resp. fast) synchronization areas and the regions where the isochrons are very close together (resp. where the gap between consecutive isochrons is important). 


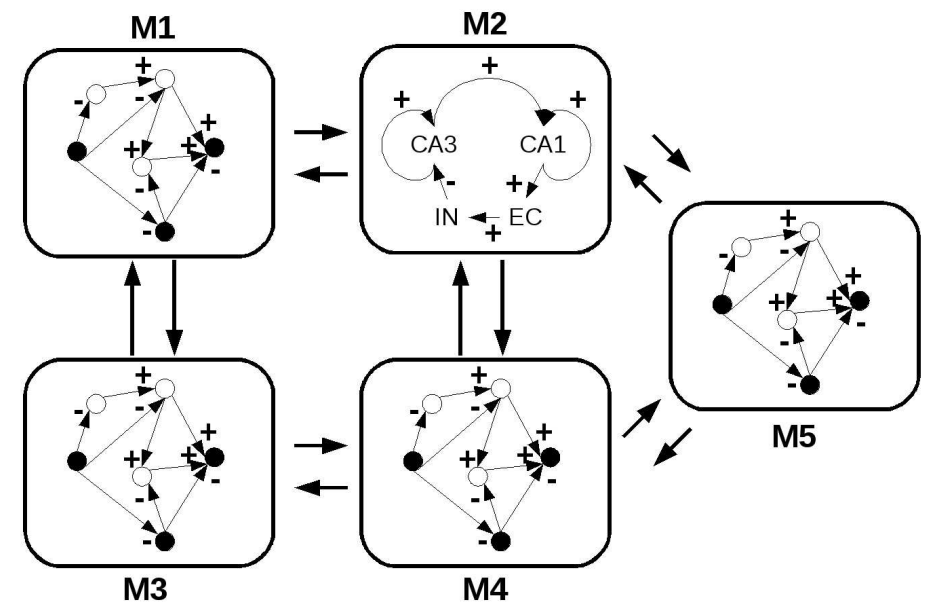

Fig. 10 Modular structure, in which the module M1 is arbitrary and M2 represents Hippocampus subnetwork.

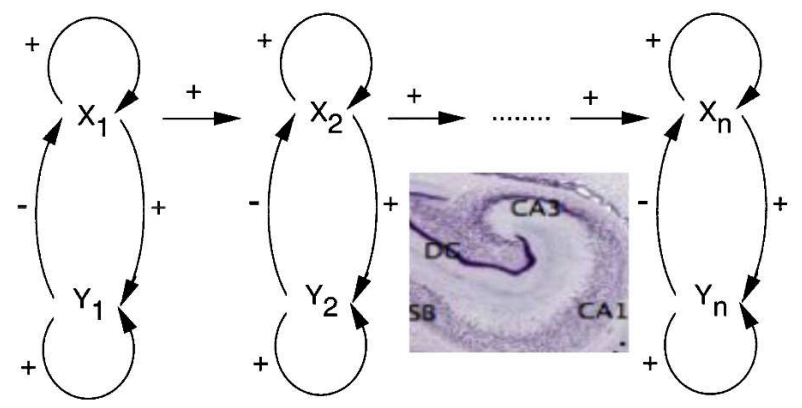

Fig. 11 Scheme of a sequential modular structure made of a chain of negative regulons, where $X_{1}$ represents the activity of CA3 and $X_{2}$ the activity of CA1 (see anatomy of CA3, CA1, DG and SB in the cartouche) 

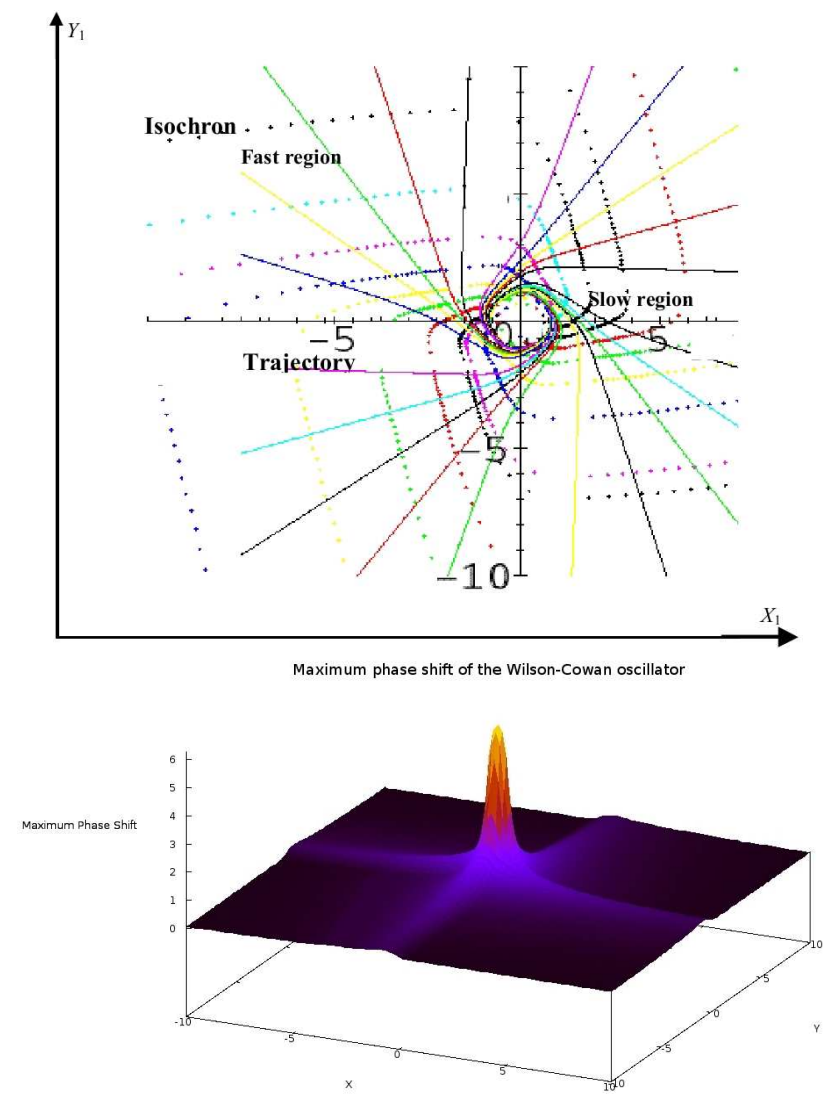

Fig. 12 (Up) Isochronal and trajectory landscape for the Wilson-Cowan dynamics of a negative regulon, showing fast (resp. slow) regions, where the velocity is high (resp. low). (Down) Fast (resp. Slow) synchronization regions in dark colors (resp. bright colors) of the Wilson-Cowan oscillator obtained by computing the maximum phase shift in response to perturbations. 


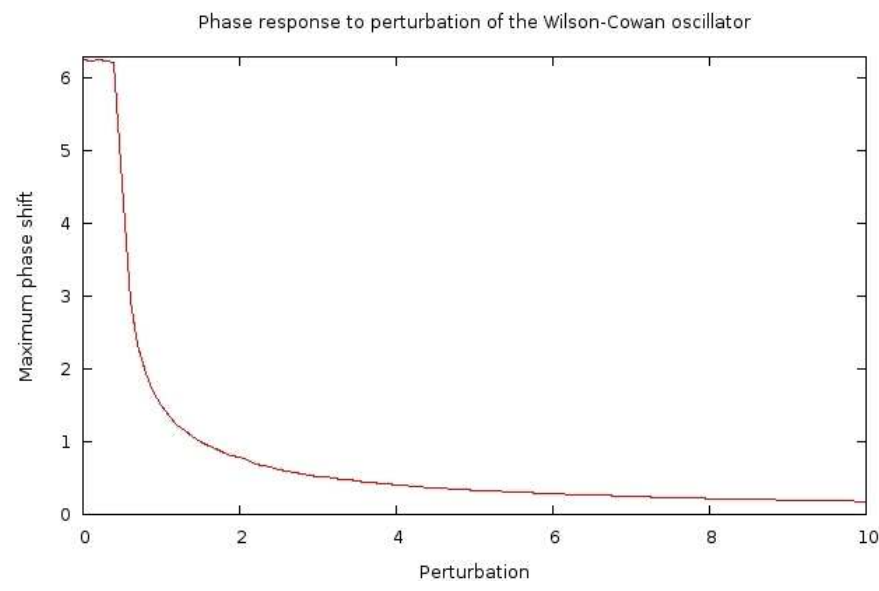

Fig. 13 The phase shift between the two isochrons containing the limit cycle after translating with respect to amplitude of perturbation. Slow (resp. fast) synchronization area are situated (qualitatively) for an amplitude below (resp. above) 2 .

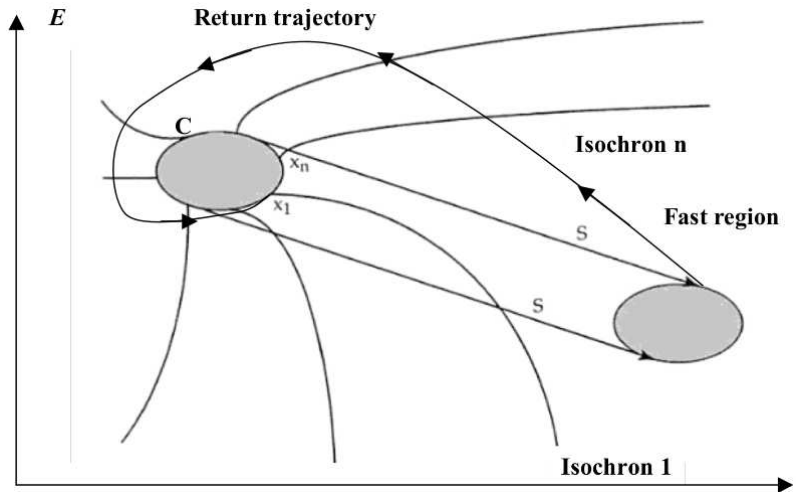

Fig. 14 Synchronizing stimulation $S$ translating the limit cycle $C$ in a fast region of the states space $E$ 


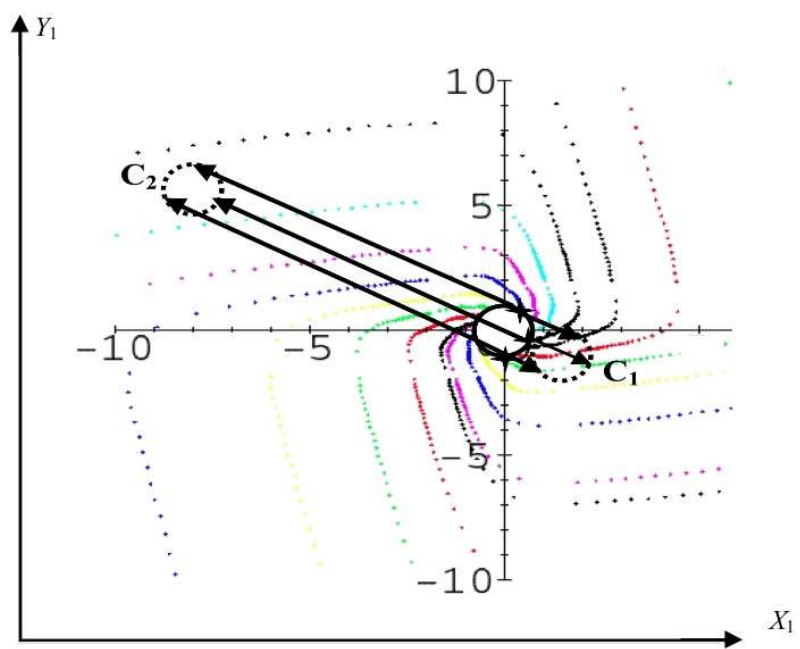

Fig. 15 Isochronal and trajectory landscape with perturbations translating the limit cycle $\mathrm{C}$ either in a desynchronizing slow region $\left(C_{1}\right)$, or in a synchronizing fast region $\left(C_{2}\right)$

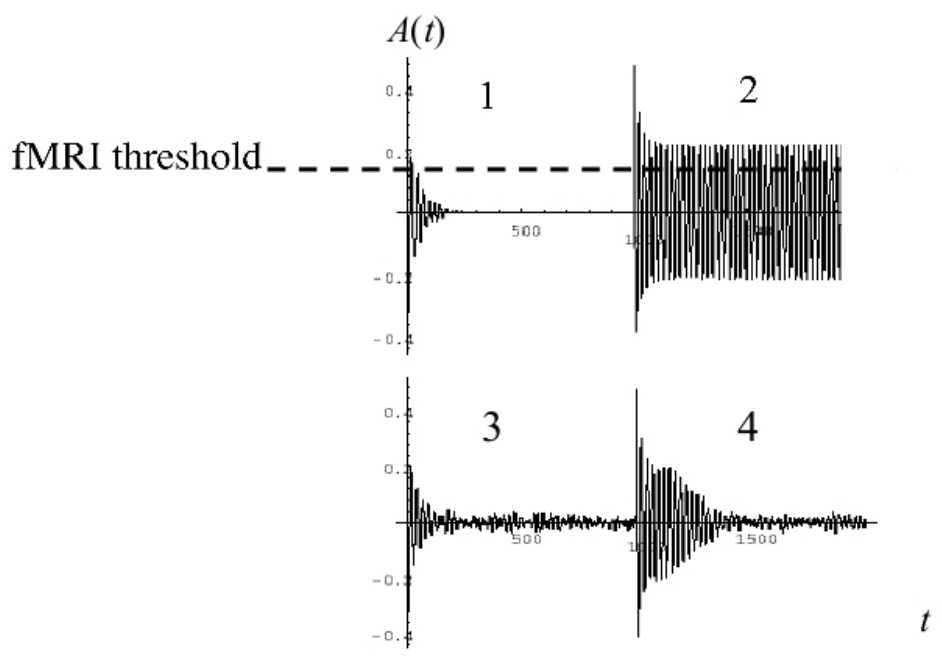

Fig. 16 Post-stimulation global activity $A(t)$ of CA3 neurons $\left(A(t)=\sum_{i=1 \ldots \ldots, n} X_{i}(t)\right)$, with a weak deterministic intra-CA3 coupling showing a fast desynchronization (1), without coupling showing a perseveration (2), with a weak noised coupling showing a fast desynchronization followed by a residual synchronization (3) and with noise without coupling showing a slow desynchronization (4) 


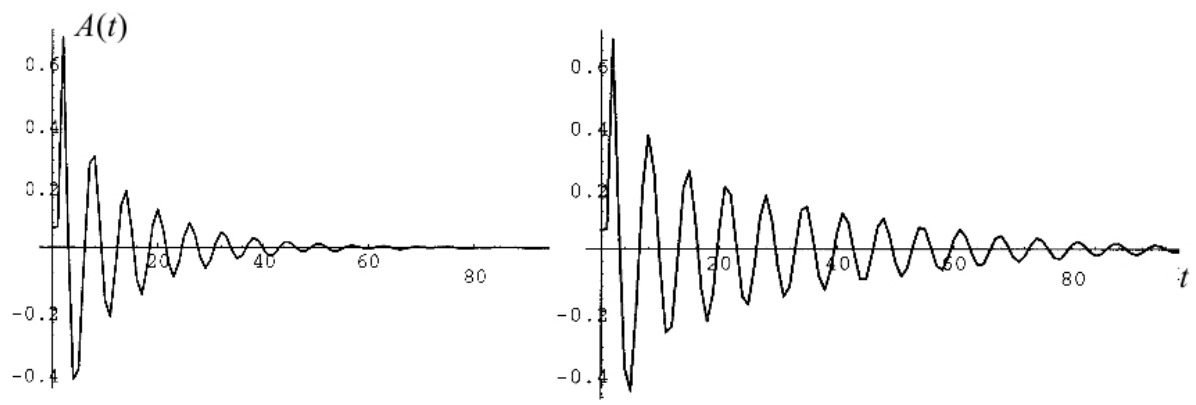

Fig. 17 Post-stimulation global activity $A(t)$ of CA3 neurons $\left(A(t)=\sum_{i=1}^{n} X_{i}(t)\right)$, (left) with a weak deterministic intra-CA3 coupling showing a fast desynchronization, and (right) with a high deterministic intra-CA3 coupling showing a slow desynchronization

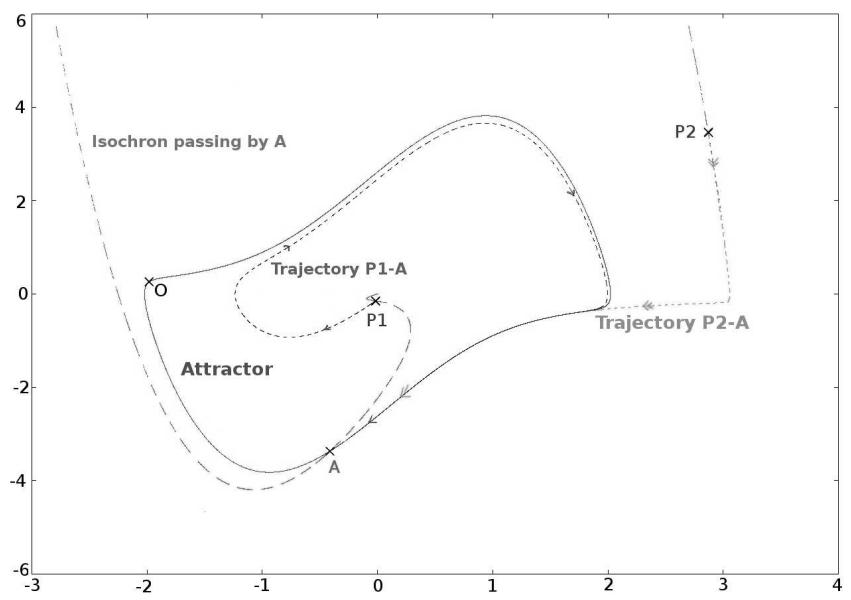

Fig. 18 Example of isochron: case of the Van Der Pol oscillator Trajectories (small dashed points) obtained from points located on both sides of the isochron (large dashed points) of phase $\phi_{A}$. The value of the phase is determined using an arbitrary reference point on the attractor (black closed curve). The period is detected when the trajectory passes again near the same point considering a threshold tolerance distance. 


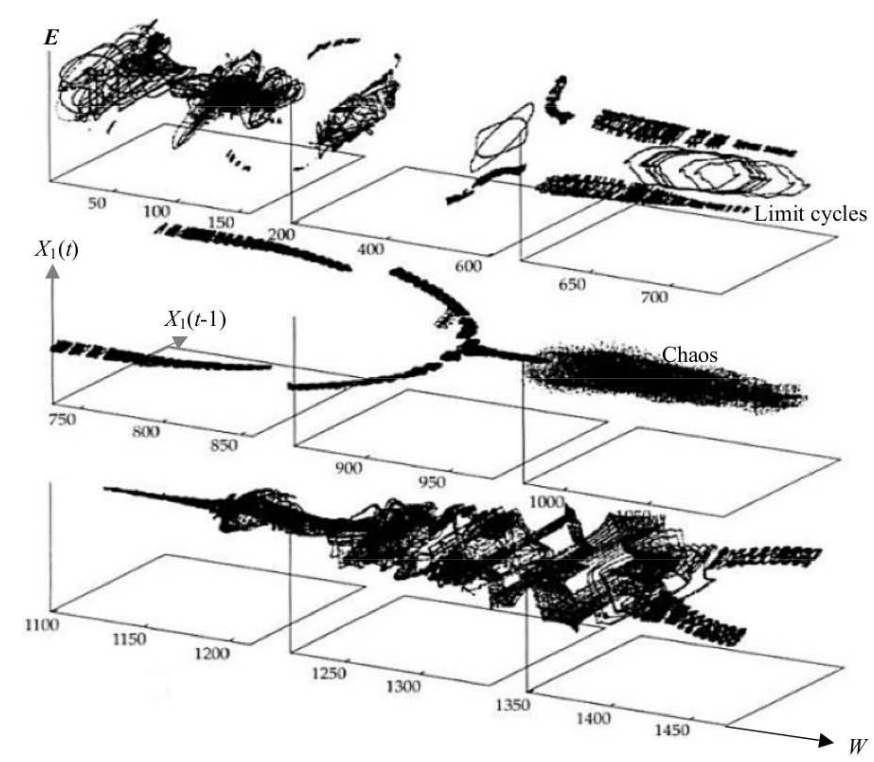

Fig. 19 Bifurcations of the attractors of the dynamics $\left(X_{1}(t-1), X_{1}(t)\right)$ for the first of 16 neurons fully connected in a classical Hopfield neural network, when a function $W$ of its synaptic weights $w_{i j}$ vary during a learning process, showing alternance of chaotic and periodic behaviours

Acknowledgements This work was supported by the Virtual Physiological Human Network of Excellence of the European Community (VPH-NoE).

\section{References}

Bartesaghi R, Migliore M, Gessi T (2006) Input-output relations in the entorhinal cortex-dentate-hippocampal system: evidence for a non-linear transfer of signal. Neuroscience 142:247-265

Ben-Amor H, Cadau S, Elena A, Dhouailly D, Demongeot J (2009) Regulatory networks analysis: robustness in morphogenesis regulation. In: AINA Workshops, 23rd International Conference on Advanced Information Networking and Applications, AINA 2009, Workshops Proceedings, Bradford, United Kingdom, May 26-29, 2009, IEEE Computer Society, pp 924-929

Bowen R (1975) $\omega$-limit sets for axiom a diffeomorphisms. J Differential Equations 18:333-339

Budelli R, Catsigeras E, Guiraud P (2010) Global dynamics of integrate and fire neural networks with instantaneous strong interactions, (in preparation)

Buzsaki G (1984) Feed-forward inhibition in the hippocampal formation. Prog Neurobiol 22:131-153

Cosnard M, Demongeot J (1985a) Attracteurs: une approche déterministe. C R Acad Sc Maths Série I 300:551-556 
Cosnard M, Demongeot J (1985b) On the definitions of attractors. Lecture Notes in Mathematics 1163:23-31

Csicsvari J, Hirase H, Czurko A, Mamiya A, Buzsaki G (1999) Fast network oscillations in the hippocampal ca1 region of the behaving rat. The Journal of Neuroscience, RC20 19:1-4

Demongeot J, Françoise JP (2006) Approximation for limit cycles and their isochrons. C R Biologies 329(12):967-970

Demongeot J, Kaufman M, Thomas R (2000) Positive feedback circuits and memory. CR Acad Sc Sciences de la Vie 323:69-79

Demongeot J, Glade N, Forest L (2007a) Liénard systems and potentialhamiltonian decomposition. i methodology. C R Acad Sci Mathématiques 344:121-126

Demongeot J, Glade N, Forest L (2007b) Liénard systems and potentialhamiltonian decomposition. ii algorithm. C R Acad Sci Mathématiques 344:191-194

Demongeot J, Ben-Amor H, Elena A, Gillois P, Noual M, Sené S (2009) Robustness in regulatory interaction networks. a generic approach with applications at different levels: Physiologic, metabolic and genetic. International Journal of Molecular Sciences 10(10):4437-4473

Dinh TP, Demongeot J, Baconnier P, Benchetrit G (1983) Simulation of a biological oscillator : the respiratory rhythm. J Theor Biol 103:113-132

Dinh TP, Demongeot J, Baconnier P, Benchetrit G (2008) A discrete time neural network model with spiking neurons. J Math Biol 56:311-345

Elena A (2009) Robustesse des réseaux d'automates booléens à seuil aux modes d'itération. application à la modelisation des réseaux de régulation génétique. PhD thesis, TIMC-IMAG, CNRS UMR5525, University JosephFourier - Grenoble, France

Elena A, Ben-Amor H, Glade N, Demongeot J (2008) Motifs in regulatory networks and their structural robustness. In: Proceedings of the 8th IEEE International Conference on Bioinformatics and BioEngineering, 2008. BIBE 2008., Athens Greece, IEEE Proceedings, pp 1-6

Forest L, Glade N, Demongeot J (2007) Liénard systems and potentialhamiltonian decomposition. applications in biology. C R Biologies 330:97106

Freire E, Gasull A, Guillamon A (2007) Limit cycles and lie symmetries. Bull Sci Maths 131:501-517

Glade N, Forest L, Demongeot J (2007) Liénard systems and potentialhamiltonian decomposition. iii applications. C R Acad Sci Mathématiques 344:253-258

Gluck MA (1996) Computational models of hippocampal function in memory. Hippocampus 6:565-566

Guckenheimer J (1975) Isochrons and phaseless sets. J Math Biol 1:259-273

Hayashi H, Nakada K, Morie T (2007) Moving object detection algorithm inspired by the sequence detection in the hippocampus and its digital LSI implementation. International Congress Series 1301:35-38 
Hefft S, Jonas P (2005) Asynchronous GABA release generates long-lasting inhibition at a hippocampal interneuron-principal neuron synapse. Nature Neuroscience 8:1319-1328

Minorsky N (1959) Theoretical aspects of nonlinear oscillations. IRE transactions on circuit theory pp 368-381

Mori M, Gahwiler BH, Gerber U (2007) Recruitment of an inhibitory hippocampal network after bursting in a single granule cell. Proc Nat Acad Sci USA 104:7640-7645

Murray JD (1993) Biological Oscillators and Switches. Springer-Verlag

Nérot O (1996) Mémorisation par forçage neuronal des dynamiques chaotiques dans les modèles connexionnistes récurrents. $\mathrm{PhD}$ thesis, Université Joseph Fourier, Grenoble

Osinga HM, Moehlis J (2009) A continuation method for computing global isochrons. Bristol Centre for Applied Nonlinear Mathematics URL http://hdl.handle.net/1983/1560

Reyes AD (2003) Synchrony-dependent propagation of firing rate in iteratively constructed networks in vitro. Nature Neuroscience 6:593-599

Ricci JCD (1995) Influence of phosphoenolpyruvate on the dynamic behavior of phosphofructokinase of escherichia coli. J Theor Biol pp 145-150

Samsonovich AV, Ascoli GA (2005) A simple neural network model of the hippocampus suggesting its pathfinding role in episodic memory retrieval. Learn Mem 12:193-208

Tonnelier A, Meignen S, Bosch H, Demongeot J (1999) Synchronization and desynchronization of neural oscillators: comparison of two models. Neural Networks 12:1213-1228

Wilson HR, Cowan JD (1974) Excitatory and inhibitory interactions in localized populations of model neurons. Biophys J 12:1-24

Winfree AT (1967) Biological rhythms and the behavior of populations of coupled oscillators. J Theor Biol 16:15-42

Winfree AT (1974) Patterns of phase compromise in biological cycles. J Math Biol 1:73-95

Winfree AT (2000) Attracting cycles and isochrons. IAM, Math. Biol. Springer 